\title{
Dislexia, déficit de atención/hiperactividad o Síndrome Irlen
}

\author{
Marcelo Bernal \\ Facultad de Psicología, Universidad de Cuenca, Cuenca, Ecuador. \\ Autor para correspondencia: marcelo.bernal@ucuenca.edu.ec \\ Fecha de recepción: 7 de mayo de 2015 - Fecha de aceptación: 7 de septiembre de 2015.
}

\begin{abstract}
RESUMEN
El objetivo de este estudio fue identificar las Distorsiones Visuales Perceptuales (DVP) y los Malestares Físicos al Leer (MFL) más comunes en los niños y niñas del tercer grado de las escuelas públicas y privadas, área urbana de Cuenca y comparar con la Tabla de Características Comunes del Síndrome Irlen, Dislexia y Déficit de Atención/Hiperactividad (TRS) propuesto por Rhonda Stone (2003). Doscientos sesenta y siete estudiantes $(n=267)$ de los terceros grados de Educación General Básica (EGB), así como directores y profesores de aula participaron en este estudio. Niños/as fueron evaluados a través de observaciones, entrevistas y la aplicación de cinco pruebas de la Escala Perceptual de Lectura Irlen (EPLI®). Medidas de frecuencia relativa (porcentajes) para las variables cualitativas y medidas de tendencia central para las variables cuantitativas fueron utilizadas. Las DVP más comunes identificadas fueron: i) necesidad de utilizar el dedo o algún marcador mientras leen $(37.59 \%)$; ii) leer lentamente o con pausas repetidas $(33.08 \%)$; iii) hacer esfuerzo para mantenerse enfocado en lo que leen, (28.95\%); iv) evitar leer o leer en voz alta $(27.89 \%)$; y v) problemas para recordar lo que se lee $(19.92 \%)$. Los MFL fueron i) acercarse o alejarse de la página (19.17\%); ii) molestias al leer bajo luces fluorescentes (18.05\%); iii) parpadeo muy seguido (14.66\%); y iv) dolor/ardor de los ojos mientras leen (12.78\%), entre otras. Al final del estudio se identificaron las DVP y MFL más comunes presentes en este grupo de estudio, se compararon con la TRS y responden a los síntomas y signos del SI propiamente y no a los de la dislexia, déficit de atención/hiperactividad.
\end{abstract}

Palabras clave: Distorsiones visuales perceptuales, malestares físicos al leer, lectura, educación básica, Síndrome Meares-Irlen, estrés visual.

\begin{abstract}
The objective of this study was to identify the most common visual perceptual distortions (VPD), and physical discomfort (PD) that affect reading in third grade children of public and private Cuenca urban schools, and compare with the table of common characteristics of Irlen Syndrome, Dyslexia, and Attention Deficit/Hyperactivity (RST), proposed by Ronda Stone (2003). Two hundred and sixtyseven third graders, as well as directors and classroom teachers participated in this study. Children were assessed through observations, interviews and applying five tests of the Irlen Reading Perceptual Scale (IRPS®). The most common visual perceptual distortions (VPD) identified were: i) the need of using the finger or marker while reading $(37.59 \%)$; ii) choppy or slow reading (33.08\%); iii) making effort to stay focused on the words (28.95\%); iv) avoiding reading or reading aloud (27.89\%), and v) having trouble remembering what was read (19.92\%). The most common physical discomforts (PD) were: i) moving closer to or further from the page $(19.17 \%)$; ii) discomfort when reading under fluorescent lights (18.05\%); iii) blinking very often (14.66\%); and iv) pain, burning of the eyes while reading $(12.78 \%)$ among others. The most common Visual Perceptual Distortions and Reading Physical Discomfort were identified for severe ranges and compared with RST. The signs and symptoms found correspond to the Irlen Syndrome rather than Dyslexia, Attention Deficit/Hyperactivity.
\end{abstract}

Keywords: Visual perceptual distortions, reading discomfort, basic education, reading difficulties, Meares-Irlen Syndrome, visual stress. 


\section{INTRODUCCIÓN}

La lectura es una de las actividades académicas más importantes que desarrolla el ser humano a través de su vida escolar. Se la considera como la actividad fundamental y de cimiento para que el resto de aprendizajes tenga lugar, pues si la lectura está ausente en el educando, puede ser ésta la causa principal de las dificultades en actividades cognitivas, psicosociales y de bienestar en general.

La Agencia Pública de Noticias del Ecuador y Suramérica (ANDES) indica que en el Ecuador, el hábito de lectura y la producción de libros no son los puntos más fuertes; que es una nación que lee poco en comparación con otros países de América Latina. En el 2012, el Centro Regional para el Fomento del Libro en América Latina y el Caribe (CERLALC, 2014) de la UNESCO, registraba una cifra de lectura en Ecuador de 0.5 libros al año por persona, que equivale a medio libro, ubicándole lejos de Chile y Argentina con 5.4 y 4.6 respectivamente, de libros leídos al año por habitante. El Instituto Nacional de Estadísticas y Censos (INEC, 2012), registró en 2013 que el 27\% de ecuatorianos no tiene el hábito de leer, de los cuales al 56.8\% no le interesa la lectura, $31.7 \%$ no leen por falta de tiempo, $3.2 \%$ por problemas de concentración y el $8.2 \%$ por otros. ANDES indica también que "otros de los problemas es que en las escuelas y colegios los docentes tampoco leen y es difícil fomentar la lectura en los niños...".

Cifras y comentarios desalentadores sobre la situación de la lectura en nuestro país nos invitan a cuestionarnos más profundamente sobre cuáles mismas son las razones de la presencia de esta problemática ecuatoriana. No se lee en Ecuador debido a la falta de interés, falta de concentración y otros aspectos que desconocemos, pero por qué esa falta de interés o de concentración? ¿Es posible acaso que la lectura sea relegada debido mas bien a la presencia de problemas de lectura desconocidos en nuestro país y no a la falta de interés en leer o a los típicos problemas de aprendizaje, y en especial los problemas de lectura o las tan llamadas dislexias, déficit de atención, hiperactividad, desinterés, fatiga visual, etc., presentes en nuestras instituciones educativas primarias, secundarias y universitarias?

Los problemas de aprendizaje más conocidos y los que más preocupan a los padres de familia, profesionales de la educación, salud y psicología a nivel mundial son los problemas de lectura o dislexias, término utilizado indistintamente, y el déficit de atención/hiperactividad. Es común escuchar a los docentes, administradores y padres de familia en general sobre la problemática de la lectura con la que se enfrentan. La mayoría de ellos indican que existe un considerable número de estudiantes que no pueden leer o que no comprenden o recuerdan nada de lo que leen, se resisten a leer, o se cansan fácilmente cuando leen, a lo cual los maestros, psicólogos, médicos y personal administrativo en especial, se apresuran en tildarles de disléxicos, hiperactivos, con problemas de déficit de atención, conducta o falta de interés generalizado por la academia. Sin embargo, el escaso conocimiento de los problemas de aprendizaje a nivel de las instituciones primarias, secundarias, inclusive universitarias y de las diferentes ramas profesionales podría ser la principal causa para un mal diagnóstico de nuestras poblaciones estudiantiles.

\subsection{Dislexia}

La Asociación Internacional de la Dislexia (2014) la define como "una dificultad de aprendizaje basado en el lenguaje. Se refiere a un grupo de síntomas los cuales ocurren en las personas con dificultades de aprendizaje con específicas habilidades de lenguaje, particularmente las de la lectura. Estudiantes con dislexia usualmente experimentan dificultades con otras habilidades como el deletreo, la escritura y la pronunciación de las palabras." Una definición formal de la lectura usada por el Instituto Nacional de la Salud del Niño y Desarrollo Humano, indica que "la dislexia está caracterizada por dificultades en la precisión o reconocimiento fluido de las palabras, y un pobre deletreo y habilidades de descodificación". Estas dificultades típicamente son el resultado de un déficit en el componente fonológico del lenguaje que a menudo no se espera que esté en relación con otras habilidades cognitivas y de la oferta de una efectiva instrucción en el aula. Las consecuencias secundarias pueden incluir problemas en la comprensión, una reducida experiencia con la lectura lo que puede impedir el aumento del vocabulario y conocimientos anteriores. Expertos actuales coinciden en que la dislexia es un trastorno basado en el lenguaje, y el tratamiento generalmente se 
centra en el uso de un enfoque de lenguaje estructurado multisensorial para ayudar a que las personas fortalezcan las vías cerebrales que conectan el habla con lo impreso. De hecho, es un nombre poco apropiado que el síntoma revelador de la dislexia sea el ver las palabras al revés. Por el contrario, la dislexia es una incapacidad de conectar las letras y palabras que se ven en la página con sus sonidos y significados. Olitsky \& Nelson (2003) define a la dislexia como la inhabilidad de desarrollar la capacidad de leer al nivel de instrucción que se espera a pesar de poseer un intelecto normal. Es un desorden primario de lectura y debe ser separado de las formas secundarias de las dificultades de lectura, las cuales incluyen al retardo mental, limitantes educacionales y ambientales, y/o enfermedades. Debido a que no existe un test estandarizado, el diagnóstico se lo realiza mediante comparaciones de las habilidades de lectura con inteligencia y estándares de lectura.

\subsection{Déficit de Atención/Hiperactividad}

Según el DMS-5 (Kupfer \& Regier 2013), el déficit de atención/hiperactividad (ADHD siglas en inglés) está caracterizado por un patrón de comportamiento, presente en múltiples escenarios como la escuela y/o el hogar, que pueden dar como resultado problemas de rendimiento a nivel social, educativo o laboral. Los síntomas están divididos en dos categorías de inatención, hiperactividad e impulsividad que incluye comportamientos de fallas de atención a los detalles, dificultades al organizar tareas y actividades, excesiva conversación, inquietud, y/o inhabilidad de permanecer sentados en situaciones apropiadas. Muchos de ellos pueden que en realidad presenten estas singularidades; sin embargo, existe la posibilidad de que al ser evaluados mediante la aplicación de pruebas de lectura, $\mathrm{u}$ otras pruebas tradicionales, estos individuos sean mal diagnosticados, puesto que presentan otro tipo de peculiaridades y necesitan diferentes instrumentos para las evaluaciones. A la vez, los docentes de aulas, requieren estrategias de intervención psicopedagógica que permitan objetivar el problema y su intervención.

Barkley (2015) define al desorden de Déficit de Atención/Hiperactividad como el estigma puesto en niños y adultos que presentan problemas significativos de atención, típicamente con impulsividad así como también una excesiva actividad. Este desorden representa una de las razones más comunes para que los niños con problemas comportamentales sean referidos a los profesionales médicos y de salud mental en los Estados Unidos y es uno de los desórdenes más prevalentes en la psiquiatría infantil. Actualmente, las referencias de adultos con ADHD está también incrementándose aceleradamente; hasta los 1990s, y aún hasta estas fechas, este grupo ha sido un segmento de la población con ADHD marcadamente desconocido y menos atendido.

\subsection{Sindrome Irlen (SI)}

Se le conoce también como Síndrome Meares-Irlen/Estrés Visual, (MISViS siglas en inglés), y Síndrome de Sensibilidad Escotópica (SSE). El Síndrome Irlen (SI) es un trastorno del procesamiento perceptual, es decir, se refiere específicamente a la forma como el cerebro procesa la información visual que recibe. No es un desorden basado en el lenguaje y la instrucción basada en la fonética no ayuda a alguien con Síndrome Irlen de la misma forma en que ayudaría a alguien con dislexia en la mejora de sus habilidades de lectura. En su esencia, el Síndrome Irlen es la sensibilidad a la luz, donde las personas son susceptibles a una determinada longitud de onda de luz la que causa los síntomas físicos y distorsiones visuales perceptuales. Estos individuos tienen dificultades de lectura no porque sus cerebros tienen problemas para conectar las letras que ven con los sonidos que éstas producen, sino porque ven distorsiones en la página impresa, o porque el fondo blanco o su resplandor molestan o irrita sus ojos, les da dolor de cabeza, o se sienten con sueño cuando intentan leer. A diferencia de la dislexia, las dificultades del Síndrome Irlen pueden ser mucho más que problemas de lectura. Las personas con Síndrome Irlen tienen dificultad para procesar toda la información visual, no sólo con la identificación de las palabras en una página impresa. A menudo estos individuos experimentan dificultades con la percepción de la profundidad, el conducir, el rendimiento deportivo, y otras áreas que no están relacionadas generalmente con la dislexia. Varias investigaciones indican que la falta de interés y/o de concentración en la lectura, problemas de entender o recordar lo que se lee, fatiga, malestares físicos al leer (MFL) y (DVP) se deben a la presencia del Síndrome Meares-Irlen/Estrés Visual (MISViS). La sintomatología del SSE puede ser 
una de las causas de los problemas en nuestro medio y pueden ser confundidos con los signos y síntomas de la dislexia, déficit de atención/hiperactividad. Irlen \& Lass (1989) define al MISViS como una disfunción perceptual la cual está relacionada con las dificultades de lectura debido a las diferentes fuentes de luz, luminosidad, intensidad, ondas de luz, y el contraste de color. Individuos que padecen de este síndrome ponen más esfuerzo en realizar sus tareas escolares y de lectura lo cual les causa fatiga por las constantes adaptaciones que deben hacer, pueden ser lectores lentos e ineficientes, tener una baja comprensión lectora, incapacidad de leer en forma continua, sufrir de fatiga o malestares de cabeza, y presentar problemas de escritura y percepción de profundidad. Dentro de las MFL del MISViS, están los dolores de cabeza, dolores de los ojos, sueño, cansancio, dificultad de concentrarse y entender lo que se está leyendo. Se han observado casos extremos de niños incapaces de permanecer quietos mientras leen, otros protestan y rechazan rotundamente leer ya que no toleran el dolor o malestar que esta actividad les causa.

La existencia del MISViS ha sido corroborada por las investigaciones realizadas por Miller (1984) y Adler \& Atwood (1987). Otros estudios reportan distorsiones similares a las que se observan en los lectores ineficientes. Jordan (1972) encontró que lectores débiles experimentaban imágenes dobles, la separación de las palabras o letras y la duplicación de las palabras. Participantes en los estudios de Stein y Fowler (1985) y Lovegrove (1984) notaban distorsiones y movimientos en lo impreso. Estudios sobre la prevalencia del MISViS en diferentes poblaciones han sido realizados a nivel mundial. Miller (1984) encontró que el $74 \%$ de adultos con problemas de aprendizaje y el 15\% de la población adulta normal o con habilidades de lectura más arriba de lo normal presentaban síntomas propios del MISViS. Robinson \& Miles (1987), Whithing (1986), Adler and Atwood (1987), e Irlen (1983) todos ellos reportan que el $46 \%$ al 50\% de los lectores débiles que participaron en sus estudios tienen síntomas de MISViS. Según Irlen, muchos individuos que padecen del MISViS son extremadamente sensitivos a ciertas frecuencias del espectro visible, el cual produce malestar bajo varias condiciones de luminosidad, acomodaciones inadecuadas del fondo de lo que se está leyendo, dificultades con la resolución de lo impreso, restringido espacio de concentración, pobre percepción de profundidad y dificultades en mantener una lectura continua. Los síntomas físicos que le acompañan pueden incluir fatiga, malestares en los ojos y dolores de cabeza.

\subsection{Tabla de características comunes del Síndrome Irlen, dislexia y déficit de atención/hiperactividad (TRS)}

Stone (2003) plantea que los signos y síntomas del Síndrome Irlen, la Dislexia, y el Deficit de Atención/Hiperactividad pueden confundirse lo que puede dar lugar a un mal diagnóstico, razón por la que propone un cuadro en donde identifica claramente cuál síntoma y/o signo las caracteriza. Recomienda que tanto profesores como padres de familias deban siempre estar vigilantes y ser los primeros en observar las diferentes manifestaciones de aquellos niños/as que tengan dificultades de aprendizaje.

Señala además que la tabla de características comunes (Tabla 1, Tabla de Rhonda Stone, TRS) puede ayudar a padres de familia a tomar decisiones con mejor información cómo proceder. Ofrece información sobre las características principales del SI, Dislexia y ADHD, y sólo se le debe tomar como referente, no reemplaza al consejo médico profesional y que muchos de estos signos y síntomas pueden entrecruzarse. Por ejemplo un niño que se vuelve físicamente incómodo al leer por quince minutos (característica común del SI) puede aparecer distraído (una característica común del Déficit de Atención/Hiperactividad). Una vez más la recomendación es observar patrones de comportamientos y no absolutos. También algunos niños reaccionarán de manera diferente a las ondas de luz u otros problemas de aprendizaje. De cualquier manera, ya sea que los niños sean afectados por la dislexia, ADHD, o el SI, éstos manifestarán varias de las características a una área correspondiente y no necesariamente a todas.

La intención de este trabajo fue identificar las DVP y MFL más comunes, síntomas propios del SI a través de la aplicación de la Escala Perceptual de Lectura Irlen (EPLI®), observaciones y entrevistas estructuradas y comparar con la TRS. Para los cálculos estadísticos fueron utilizadas las medidas de frecuencia relativa (porcentajes) para las variables cualitativas y medidas de tendencia central para las variables cuantitativas. Una vez obtenidos los resultados se hicieron las 
comparaciones que ofrece la literatura con los síntomas y signos de la dislexia, déficit de atención/hiperactividad. La contribución de este estudio es el de dar a conocer que la presencia de dificultades de lectura, el desinterés por leer, presencia de DVP y MFL, entre otros, no necesariamente pueden responder a la dislexia, hiperactividad, o déficit de atención sino al Síndrome Irlen, (SI).

Tabla 1. Tabla de Características Comunes del Síndrome Irlen, Dislexia, Déficit de Atención/ Hiperactividad (Tabla de Rhonda Stone (TRS), 2003).

\begin{tabular}{rrrr}
\hline Característica & Síndrome Irlen & Dislexia & ADHD \\
\hline FÍSICAMENTE OBSERVABLE & & & \\
Arden lo ojos, pican, o se ponen llorosos & $\checkmark$ & & \\
Experimenta dolores de cabeza, del estómago, o & $\checkmark$ & $\checkmark$ & $\checkmark$ \\
fatiga & & & \\
Se frota los ojos frecuentemente & $\checkmark$ & & \\
Hace sombras para sus ojos mientras lee & $\checkmark$ & \\
Bizquea o parpadea excesivamente & $\checkmark$ & \\
Inclina su cabeza para leer & $\checkmark$ & \\
Físicamente se fatiga rápidamente durante la lectura (15') & $\checkmark$ & \\
Lee bajo luces tenues & $\checkmark$ & \\
Historia familiar de malestares con la lectura, sensibilidad a & $\checkmark$ & \\
la luz, dolores de cabeza o migraña & $v$ & \\
\hline
\end{tabular}

\section{PATRONES DE LECTURA}

Se salta palabras o líneas enteras durante la lectura

Dificultad al pronunciar las palabras y mezclar

Fue lento al aprender a hablar

Es un lector lento

La comprensión es pobre

Evita leer o toma frecuentes recesos

\section{PROBLEMAS DE PERCEPCIÓN VISUAL}

Ve lo impreso como borroso o inestable

Confunde números en problemas de matemáticas

Percibe distorsiones visuales en la página impresa

Lee palabras que están al revés

Experimenta yuxtaposición de letras

Dificultades en aprender a decir la hora

\section{PROBLEMAS DE PERCEPCIÓN AUDITIVA}

Lento para aprender la conexión entre letras individuales y sonidos

Dificultad al pronunciar las palabras y mezclar palabras

Fue lento al aprender a hablar

Tiene dificultades para seguir instrucciones verbales

Habla excesivamente

\begin{tabular}{lll}
$v$ & $v$ & $v$ \\
$v$ & $v$ & $v$ \\
$v$ & $v$ & $v$ \\
$v$ & $v$ & $v$ \\
$v$ & $v$ & $v$ \\
$v$ & $v$ & $v$ \\
\hline
\end{tabular}

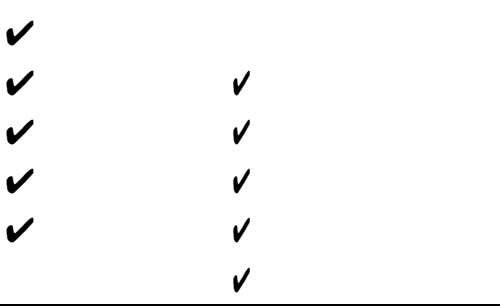

$\begin{array}{ll}v & \\ v & \\ v & \\ v & v\end{array}$

IMPRESIONES/LETRA MANUSCRITA

Problemas relacionados con la disgrafia

Desigualdad en el tamaño de la letra o en el espaciado

Dificultad de escribir en las líneas de la hoja

Letra manuscrita deficiente, evita escribir en cursiva

Extraña forma de agarrar el lápiz o esfero

Predispuesto a la escritura en espejo (escribir en reversa)

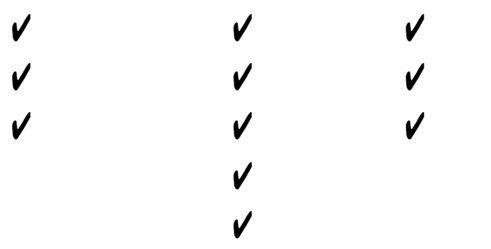




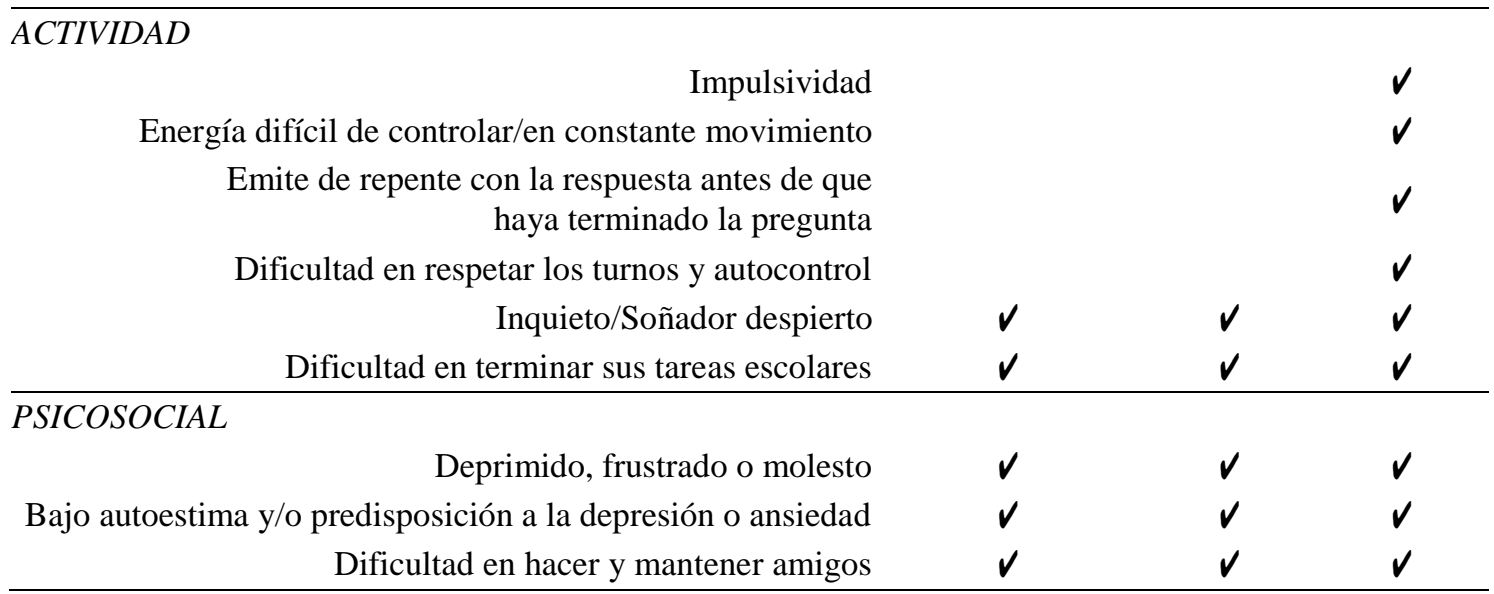

Tomado de The Light Barrier, Rhonda Stone (2003), pp. 134-136, traducido por el autor.

Nuestros datos indican que existen síntomas y signos parecidos a los problemas de aprendizaje más comunes en las escuelas urbanas de Cuenca. Varios estudios sobre la temática recomiendan no hacer diagnósticos apresurados que no tengan sólidos soportes científicos, y en el caso particular de problemas de aprendizaje, Irlen plantea que el $46 \%$ más bien pueden responder a la sintomatología del SI. Se recomienda por lo tanto, que antes de administrar las diferentes pruebas de rutina en las escuelas, colegios y universidades, centros médicos, de recuperación escolar, etc., se consulte en primera instancia con el oftalmólogo, u optometrista para un examen visual completo y se corrija los problemas visuales previos a la de detección del Síndrome Irlen. Al contar con los resultados de los exámenes visuales de rutina y al eliminar las DVP y los MFL estaríamos descartando aquellas barreras físicas y perceptuales que impiden que se dé la lectura, que en su mayoría es la base de las pruebas administradas a aquellos niños que presentan dificultades de aprendizaje.

\section{MATERIALES Y MÉTODOS}

\subsection{Población de estudio}

En esta investigación se trabajó con niños del tercer grado de educación general básica (EGB), de las quince (15) escuelas del casco urbano de la ciudad de Cuenca, provincia del Azuay-Ecuador, con una muestra de 267 estudiantes durante el segundo quimestre, febrero-julio e inicios de septiembre del año escolar 2013-2014. Es un estudio descriptivo, transversal de corte, básico que planteó identificar las distorsiones visuales perceptuales (DVP) y los Malestares Físicos al Leer (MFL) más comunes en los niños y niñas del tercer grado de las escuelas públicas y privadas, área urbana de Cuenca y comparar con la Tabla de Características Comunes del Síndrome Irlen, Dislexia y Déficit de Atención/Hiperactividad (TRS) (Stone, 2003).

\subsection{Población, muestreo y muestra}

Según los archivos Excel de establecimientos educativos, Distrito 1, proporcionado por el Ministerio de Educación del Ecuador, del Cantón Cuenca, Provincia del Azuay, Zona 6, en diciembre del 2013, la población de estudiantes del tercer grado de educación básica de las escuelas del casco urbano fue de 6.638 niños/as. Para el muestreo utilizamos el Software para iOS Mi muestra v.1.0 y se determinó que la muestra con un Nivel de Confianza (NC): del $99 \%$ y un margen de Error (M.E.) del $4.36 \%$ fue de 262; referente internacional de MISViS fue la proporción del 12\%. La selección de los niños/as para la aplicación de las pruebas en las escuelas se la hizo a través de la aleatorización con la aplicación móvil "Random Number" y la selección manual aleatoria en los listados de los terceros grados entregados por los establecimientos educativos. El trabajo de investigación se enfocó en el trabajo con los niños/as que no presentan ninguna sintomatología física, cuya visión es normal, usen o no lentes o los que usan lentes al momento de tomar las pruebas y que no sufren de ningún 
trastorno visual que demanda atención constante, pero se excluyeron a aquellos escolares que tienen problemas físicos visuales como cataratas, ceguera de un ojo, etc., o algún tipo de trastorno emocional o psicológico o los que tenían deficiencia visual y no estaban usando lentes al momento de la aplicación de las pruebas de la EPLI®.

\subsection{Instrumento para la recolección de la información}

Para la recolección de datos se utilizó la EPLI ${ }^{\circledR}$, que contiene 4 secciones. Sección 1 recoge datos sobre las dificultades y malestares al leer en una escala de: no aplicable rango cuantitativo (rc) 0 , leve, rc1-3, moderado, rc.4-7 y severo, rc 8-17. En la Sección 2, se anotan las observaciones y la información al realizar las pruebas de Cubo A, Cubo B y de la Calabaza(s) que son pruebas para corroborar lo manifestado en la Sección 1. También se registran los síntomas físicos al realizar las pruebas de límite de enfoque y la prueba de señalar. En la Sección 3 se anota el color de la(s) transparencia(s) tanto para el individuo sujeto de la investigación y si es posible, la selección del color de transparencia que hizo alguno de los niños. Adicionalmente en esta sección se anotarán las reacciones del sujeto al leer material en hojas de color blanco y el grado de mejoramiento con las transparencias de color en escalas de no aplicable N/A, ligero, moderado, considerable. En la Sección 4 se registra la información al aplicar las páginas de distorsiones del Manual Irlen, así como también los comentarios relevantes a tomarse en cuenta durante la evaluación. Esta hoja de datos viene con las correspondientes escalas referenciales o baremos de cada sección para obtener datos cuantitativos. Los resultados de las pruebas fueron corroborados mediante las observaciones de la sintomatología y los signos propios en los niños que padecen el MISViS.

\subsection{Procedimiento de recolección de la información}

Cada niño/a se entrevistaba con el evaluador quien en primera instancia recogía datos personales del alumno, luego se hacía las 17 preguntas de la Secciones 1 y 2 de la EPLI®. Si el entrevistado contestaba un (x) número de respuestas, se procedía a marcar en los correspondientes baremos, escalas leve, moderado, y severo. También se administraron las pruebas de la Sección 2, prueba del Cubo A, Cubo B, Calabaza(s), y las pruebas de apuntar, con el fin de comprobar lo que los niños/as indicaban en la Sección 1. Para la culminación de las pruebas, en algunas ocasiones por motivos de asegurar la validez de las mismas, se pedía al entrevistado que leyera un texto correspondiente al tercer grado de básica ya sea antes o después de las pruebas.

\subsection{Procedimiento de análisis de los datos}

El registro de la información proporcionada por los evaluados fueron anotados directamente en la hoja de datos de la EPLI ${ }^{\circledR}$, al igual que las observaciones adicionales. Al responder al cuestionario 1 y 2, el investigador escribía las respuestas emitidas por el evaluador en el caso de detectar los signos y síntomas del MISViS, contaba el número de respuestas y marcaba el puntaje obtenido de las 17 preguntas tanto de las DVP y MFL en los respectivos rangos de valoración de 0 a 17 . Para efectos de procesamiento de datos, se tomó en consideración las 4 escalas de apreciación de la presencia del Síndrome MISViS. (No Irlen 0, Leve 1-3, Moderado 4-7, Severo 8-17). Los datos obtenidos en las hojas de datos EPLI ${ }^{\circledR}$, fueron subidos al programa SPSS 21; se formó la base de datos para ser tratados de acuerdo a las diferentes variables, correspondientes a cada ítem del cuestionario de preguntas sobre las DVP y MFL. El análisis estadístico se realizó mediante medidas de frecuencia relativa (porcentajes) para las variables cualitativas y medidas de tendencia central para las variables cuantitativas.

\section{RESULTADOS}

De las 17 DVP mencionadas en la Sección 1, de la EPLI ${ }^{\circledR}$, las más comunes, se observan detalladamente en la Tabla 2. Se presentan en un rango severo, las siguientes: i) los escolares 
utilizaron su dedo o un marcador para leer $(37.59 \%)$; ii) leen lentamente y con pausas repetidas (33.08\%); iii) hacen esfuerzo para mantenerse enfocados en las palabras que leen (28.95\%); iv) $27.89 \%$ evitan leer en voz alta; v) tienen problemas para recordar y entender lo que leen $(19.92 \%$ y $14.66 \%$ ) respectivamente; vi) $16.54 \%$ de estos escolares se ponen inquietos, hiperactivos y nerviosos; vii) se distraen, toman repetidos descansos mientras realizan las pruebas (17.29\%); viii) repiten las líneas de abajo o de arriba del texto que se lee (14.66\%); y ix) se pierden de lugar cuando están leyendo $(10.15 \%)$.

Tabla 2. Distorsiones Visuales más comunes.

\begin{tabular}{cll}
\hline \multicolumn{3}{l}{ Distorsiones Visuales Perceptuales más comunes N (\%) } \\
\hline 1 & Usa el dedo o algún marcador para leer & $100(37.59)$ \\
2 & Lee lentamente o con pausas repetidas & $88(33.08)$ \\
3 & Hace esfuerzo para mantenerse enfocado en lo & $77(28.95)$ \\
& que lee & $74(27.89)$ \\
4 & Evita leer o leer en voz alta & $53(19.22)$ \\
5 & Tiene problemas para recordar lo que lee & $46(17.29)$ \\
6 & Se distrae o toma repetidos descansos & $44(16.54)$ \\
7 & Se pone activo/a, inquieto/a o nervioso/a & $39(14.66)$ \\
8 & Tiene problemas para entender lo que lee & $39(14.66)$ \\
9 & Por accidente repite o vuelve a leer las líneas & $27(10.15)$ \\
10 & Pierde su lugar & \\
\hline
\end{tabular}

De los 17 MFL, Sección 1 de la EPLI ${ }^{\circ}$, los más comunes presentes en los sujetos de investigación de ambos sexos en una escala de siempre se encuentran detallados en la Tabla 3. De mayor a menor porcentaje se destacan los siguientes: i) los escolares tienen necesidad de alejarse o acercarse al material que leen o al realizar las pruebas de la EPLI® $(19.17 \%)$; ii) molestias de la lectura bajo luces fluorescentes (18.05\%); iii) abren sus ojos exageradamente cuando están leyendo o realizando las pruebas Irlen (14.66\%); iv) parpadeo continuo (14.66\%); v) sus ojos se sienten secos, ásperos, les pican, les duelen o les arden (12.78\%); vi) $12.41 \%$ de los escolares se frotan sus ojos o sus alrededores al realizar las pruebas; vii) el $11.28 \%$ se pone inquieto/a, activo/a o nervioso/a al leer; y viii) fruncen el ceño o bizquean (10.53\%).

Tabla 3. Malestares físicos al leer más comunes.

\begin{tabular}{lll}
\hline & Malestares físicos al leer más comunes & $\mathrm{N}(\%)$ \\
\hline 1 & Se acerca o se aleja de la página & $51(19.17)$ \\
2 & Le molesta leer bajo luces fluorescentes & $48(18.05)$ \\
3 & Abre sus ojos exageradamente & $39(14.66)$ \\
4 & Parpadea muy seguido & $39(14.66)$ \\
5 & Se sienten secos, ásperos o picosos & $34(12.78)$ \\
6 & Le duelen o le arden & $34(12.78)$ \\
7 & Se frota los ojos o alrededor de los ojos & $33(12.41)$ \\
8 & Se pone inquieto/a, activo, nervioso/a & $30(11.28)$ \\
9 & Frunce el seño o bizquea & $28(10.53)$ \\
\hline
\end{tabular}

Tabla 4 indica cuáles son las DVP y MFL más comunes y los compara con la sintomatología de la Dislexia y el Déficit de Atención/Hiperactividad propuesta por Stone (2003). Los resultados de nuestro estudio revelan la presencia de la sintomatología propia del SI, DVP (perderse de lugar mientras se lee, volver a repetir lo leído, el evitar leer en voz alta, problemas de comprensión); MFL (ojos secos, ásperos y picosos, fruncir el ceño o bizqueo, molestias bajo luces fluorescentes). 
Tabla 4. Características del SI, DVP y MFL más comunes, Dislexia, ADHD.

\begin{tabular}{|c|c|c|c|c|}
\hline Característica & SI & $\begin{array}{l}\text { DVP } \\
\text { MFL }\end{array}$ & Dislexia & ADHD \\
\hline Arden lo ojos, pican, o se ponen llorosos & $\checkmark$ & $V$ & & \\
\hline Se frota los ojos frecuentemente & $\checkmark$ & $\checkmark$ & & \\
\hline Bizquea o parpadea excesivamente & $\checkmark$ & $\checkmark$ & & \\
\hline $\begin{array}{l}\text { Historia familiar de malestares con la lectura, } \\
\text { sensibilidad a la luz, dolores de cabeza o } \\
\text { migraña }\end{array}$ & $\checkmark$ & $\checkmark$ & & \\
\hline $\begin{array}{l}\text { Se salta palabras o líneas enteras durante la } \\
\text { lectura }\end{array}$ & $\checkmark$ & $\checkmark$ & $\checkmark$ & $\checkmark$ \\
\hline Pierde de lugar en la lectura fácilmente & $V$ & $\checkmark$ & $\checkmark$ & $\checkmark$ \\
\hline Es un lector lento & $\checkmark$ & $\checkmark$ & $\checkmark$ & $\checkmark$ \\
\hline La comprensión es pobre & $\checkmark$ & $\checkmark$ & $\checkmark$ & $\checkmark$ \\
\hline Evita leer o toma frecuentes recesos & $\checkmark$ & $\checkmark$ & $\checkmark$ & $\checkmark$ \\
\hline Inquieto/Soñador despierto & $\checkmark$ & $\checkmark$ & $\checkmark$ & $\checkmark$ \\
\hline Dificultad en terminar sus tareas escolares & $v$ & $v$ & $v$ & $v$ \\
\hline
\end{tabular}

\section{DISCUSIÓN}

El propósito de este estudio fue el de identificar las DVP y los MFL más comunes del Síndrome Irlen y compararlos con la TRS, debido a que durante la investigación la mayoría del personal docente y administrativo de las escuelas participantes manifestaron desconocer del Síndrome Irlen y más bien confundían su sintomatología con las de la dislexia, hiperactividad, déficit de atención entre otras. Afirmaban conocer de sus sintomatologías; sin embargo, estos síntomas pueden ser confundidos con los del SI ya que es particularmente muy prevalente en las personas que tienen dificultades de lectura, tales como la dislexia pero puede darse también en los buenos lectores. Irlen et al. (1991), Conlon et al. (1999), Kriss \& Evans (2005) y Kruck et al. (2008) plantean que aunque originalmente se pensó que el SI era una sub-clasificación de las personas que sufren dislexias. Investigaciones más recientes han comenzado a diferenciar a estas dos patologías y sustentar que el MISViS y las dificultades de lectura pueden ser independientes. Los diagnósticos apresurados emitidos a niños y adultos que presentan similitudes de las tres patologías mencionadas en la TRS por profesores de aula, médicos, psicólogos pueden confundir a padres de familia, familiares, etc., al mismo estudiante y utilizarse equivocadas alternativas de tratamiento o remediación. En la mayoría de casos las terapias aplicadas en las correcciones de dificultades de aprendizaje no dan los resultados esperados puesto que los signos y síntomas pueden en ocasiones responder a las tres patologías. La TRS indica que el experimentar dolores de cabeza, del estómago, o fatiga; saltarse palabras o líneas enteras durante la lectura; perderse de lugar en la lectura fácilmente; ser un lector lento; tener una comprensión pobre; evitar leer o tomar frecuentes recesos corresponden tanto al SI, Dislexia como al ADHD. Igualmente, la desigualdad en el tamaño de la letra o en el espaciado; dificultad de escribir en las líneas de la hoja; letra manuscrita deficiente, y evitar escribir en cursiva; estar inquieto/soñar despierto; dificultad en terminar sus tareas escolares; encontrarse deprimido, frustrado o molesto; bajo de autoestima y/o predisposición a la depresión o ansiedad y dificultad en hacer y mantener amigos son características de estas tres patologías.

Las DVP (10) y MFL (9) más comunes identificadas en nuestro estudio coinciden con lo que se muestran en la TRS. Se observa entonces que esta sintomatología, concuerda plenamente con las del SI, ratificándose de esta manera que pueden dar lugar a malinterpretaciones, malos diagnósticos, y causar daños físicos emocionales, y psicosociales en general en los evaluados. Por lo tanto, para tratar los casos de dificultades de aprendizaje, en especial los problemas de lectura, Dislexias, Déficit de Atención/Hiperactividad se recomienda: i) contar con las evaluaciones oftalmológicas de rutina; ii) tomando en consideración la prevalencia del Síndrome Irlen en nuestro medio escolar, $(25.84 \%)$, 
casos severos (Bernal, 2015), realizar la evaluación Irlen como segunda opción con el fin de eliminar las DVP y MFL en parte o en su totalidad; iii) proceder a las evaluaciones de Dislexias, Déficit de Atención/Hiperactividad; y iv) hacer las comparaciones respectivas con la TRS. Este procedimiento nos capacitará para la identificación más clara y la diferenciación precisa de estas tres patologías mencionadas. Los equipos multidisciplinarios trabajarán más efectivamente en la detección de los diferentes problemas de aprendizaje en nuestro medio escolar. El tener la TRS, posibilita hacer comparaciones de diagnósticos más precisos lo que permite seleccionar el uso de las adecuadas terapias y tratamientos de los trastornos de aprendizaje, ahora mejor identificados.

\section{CONCLUSIONES}

Se identificaron las DVP más comunes en los escolares del tercer grado de las escuelas del área urbana de Cuenca las cuales se resumen: i) pierden el lugar donde están leyendo; ii) por accidente repiten palabras; iii) evitan leer o leer en voz alta; iv) leen lentamente o con pausas repetidas, entre otras. Asimismo se identificaron los MFL: i) dolor o ardor de los ojos; ii) sentir sus ojos secos, ásperos o picosos; iii) molestias de leer bajo luces fluorescentes; entre otros, y se compararon las DVP y MFL más comunes del estudio con la TRS y se encontraron las siguientes similitudes entre SI, Dislexia, Déficit de Atención/Hiperactividad: i) ardor de los ojos, picazón, o llorosos; ii) frecuente restriegue de los ojos; iii) excesivo bizqueo o parpadeo; iv) sensibilidad a la luz, dolores de cabeza o migraña; v) salto de palabras o líneas enteras durante la lectura, entre otras. Concluimos indicando que es necesario: i) utilizar los resultados de la presente investigación para planificar estrategias de evaluación educativa dirigidas a reducir diagnósticos apresurados de las dificultades de aprendizaje; ii) realizar nuevas investigaciones con igual metodología para la identificación del Síndrome Irlen; y iii) implementar un sistema de evaluación en las escuelas que sigan el siguiente protocolo: a) Evaluaciones oftalmológicas y optométricas de rutina; b) Evaluación Irlen con el fin de eliminar las barreras que impiden la objetividad en las evaluaciones en las patologías de la Dislexia, Déficit de Atención/Hiperactividad; c) Evaluaciones de Dislexia, Déficit de Atención/ Hiperactividad; d) implementar programas de intervención en los diferentes establecimientos educativos con el apoyo multidisciplinario de profesionales médicos, educadores, psicólogos y administradores; y, e) ampliar las investigaciones sobre el Síndrome y Método Irlen a nivel local, regional y nacional.

\section{AGRADECIMIENTOS}

Mis agradecimientos a la Dra. Sandra Tosta, Directora de Investigación del Instituto Irlen y al Dr. Jorge García, asesores estadísticos del proyecto, al Dr. Fernando Estévez y Mgst. Felipe Webster, investigadores de la Universidad de Cuenca, a los miembros del club de Neurociencias de la Facultad de Medicina y al club de Neuropsicología de la Facultad de Psicología por su colaboración en los trabajos de campo, a David Tacuri, ayudante principal de investigación, por la coordinación y el apoyo continuo en el desarrollo de la investigación. Mi especial gratitud al Dr. Jan Feyen por su valiosa contribución científica en la revisión, corrección y aporte a este estudio desde sus inicios.

\section{REFERENCIAS}

Adler, L., M. Atwood, 1987. Poor Readers: What do they really see on the page? Study produced by the East San Gabriel Valley Regional Occupational Program in Cooperation with the California Department of Education, Youth, Adult and Alternative Educational Service Division. 
Asociación Internacional de la Dislexia, 2014. Do I Have Dyslexia or Irlen Syndrome?

Disponible en http://irlen.com/the-difference-between-irlen-syndrome-and-visual-dyslexia/.

Bernal, M., 2015. Prevalencia del Síndrome Meares-Irlen/Estrés Visual que afecta la lectura en niños de tercer grado. Facultad de Psicología, Universidad de Cuenca, Cuenca. MASKANA, 6(1), 69-78.

CERLALC, 2014. Centro Regional para el Fomento del Libro en América Latina y el Caribe de la UNESCO. Día Mundial del Libro: Ecuador mantiene un bajo hábito de lectura. Disponible http://www.andes.info.ec/es/noticias/dia-mundial-libro-ecuador-mantiene-bajo-habitolectura.html.

Conlon, E.G., W.J. Lovegrove, E. Chekaluk, P.E. Pattison, 1999 Measuring visual discomfort. Vis. Cogn., 6(6), 637-663.

INEC, 2012. Hábitos de lectura en Ecuador. Instituto Nacional de Estadísticas y Censos. Disponible en http://www.inec.gob.ec/documentos_varios/presentacion_habitos.pdf, 14 pp.

Irlen, H., M.J. Lass, 1989. Improving reading problems due to symptoms of scotopic sensitivity syndrome using Irlen Lenses and overlays. Education, 109(4), 413-417.

Irlen, H.,1983. Successful treatment of learning disabilities. Paper presented at the 91st annual Convention of the American Psychological Association, Anaheim, CA, USA.

Irlen, H., G.R. Mettam, L.B. Adam, 1991. How to prepare an electronic version of your article. In: Jones, B.S., S.Z. Smith (Eds.). Introduction to the electronic age. New York: E-publishing Inc., 281-304.

Jordan, D.R., 1972. Dyslexia in the classroom. Chicago, Illinois: Bell \& Howell Co.

Kriss, I., B.J.W. Evans, 2005. The relationship between dyslexia and Meares-Irlen syndrome. J. Res. Read., 28(3), 350-364.

Kupfer, D., D. Regier, 2013. Diagnostic and statistical manual of mental disorders $\left(5^{\text {th }}\right.$ ed.). American Psychiatric Association, Disponible en http://psychiatry.org/psychiatrists/practice/dsm.

Kruck, R., K. Sumbler, D. Willows, 2008. Visual processing of children with Meares-Irlen syndrome. Ophthalmic Physiol Opt., 28(1), 35-46.

Lovegrove, W., 1984. Dyslexia and the visual factor. Education News, 19, 15-18.

Miller, L.,1984. Scotopic sensitivity and reading disability. Unpublished Master of Education thesis, California State University, CA, USA.

Olitsky, S.E., L.B. Nelson, 2003. Reading disorders in children. Pediatric Clinics of North America, 50(1), 213-224.

Robinson, G.L., J. Miles, 1987. The use of colored overlays to improve visual processing: A preliminary survey. The Except. Child., 34(1), 65-70.

Barkley, R.A., 2015. Attention deficit hyperactivity disorder. A Handbook for Diagnosis \& Treatment ( $4^{\text {th }}$ ed.). New York: The Guilford Press, NY, USA.

Stein, J., S. Fowler, 1985. Effect of monocular occlusion on viso-motor perception and reading in dyslexic children. Lancet, 2(8446), 69-73.

Stone, R., 2003. The light barrier. Understanding the mystery of Irlen syndrome and light based reading difficulties $\left(1^{\text {st }}\right.$ ed.). New York: Ed. St. Martin's Griffin, NY, USA.

Whithing, P., 1985. How difficult can reading be? New insight into reading problems. The Teaching of English. J. English Teachers' Assoc., 49, 49-55. 